\title{
Síndrome do trefinado: relato de caso
}

\section{Syndrome of Trephined: A Case Report}

\author{
Augusto Santos ${ }^{1}$ Diego Oliveira da Mata ${ }^{2}$ Rayane Toledo Simas ${ }^{2}$ Rodrigo Moreira Faleiro ${ }^{3}$ \\ Thiago Oliveira Lemos de Lima ${ }^{4}$
}

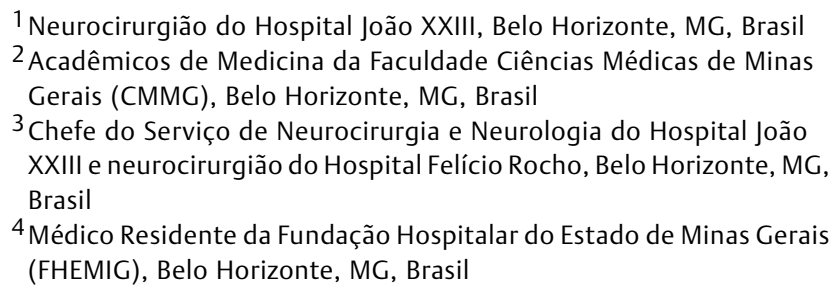

Address for correspondence Rayane Toledo Simas, MS 4y, Rua Paineiras 1117, Eldorado, Contagem, MG, Brasil CEP: 32310-400 (e-mail: raratosi@gmail.com).

Arq Bras Neurocir 2015;34:327-330.

\section{Resumo \\ Palavras-Chave \\ - craniectomia descompressiva \\ - traumatismos encefálicos \\ - síndrome}

Síndrome do trefinado é atualmente uma complicação comum na neurotraumatologia, sendo descrita como uma síndrome na qual ocorre deterioração neurológica acompanhada de sinais e sintomas após a remoção de uma parte considerável de osso do crânio, assim como ocorre na hemicraniectomia. Neste artigo, juntamente com a revisão de literatura, será relatado o caso de um paciente adulto, vítima de acidente automobilístico, com história de traumatismo cranioencefálico (TCE) grave que foi submetido à craniectomia terapêutica, cursando com a síndrome do trefinado.

"Syndrome of the Trephined" or "Sinking Skin Flap Syndrome" is an usual syndrome in which neurological deterioration occurs following removal of a large skull bone flap (for example, in descompressive craniectomy). In this article, we will report the case of a 24 years old male, victim of an automobile accident with severe traumatic brain injury (TBI), which developed the Syndrome of the Trephined.

\section{Introdução}

Síndrome do trefinado (ST) é uma síndrome em que ocorre deterioração neurológica após a remoção de uma parte considerável do crânio. ${ }^{1,2}$ A ST é frequentemente não diagnosticada, e seus sintomas são melhorados após a cranioplastia. Conceitualmente, essa síndrome pode ser definida como a conversão de uma caixa fechada (closed box) para uma caixa aberta (open box). ${ }^{2}$

Assim, reportaremos o caso de um adulto jovem, vítima de trauma automobilístico, submetido à craniectomia, seguido de complicações da ST. received

November 10, 2013

accepted

August 7, 2015

published online

October 7, 2015

\section{Relato de Caso}

Paciente homem de 24 anos de idade, vítima de acidente automobilístico em novembro de 2013, com história de craniectomia bifrontotemporal devido a TCE grave. Evoluiu bem, apresentando-se apenas confuso na alta hospitalar, sendo programada cranioplastia eletiva posterior. Dois meses depois cursou com rebaixamento do sensório e abaulamento da área de craniectomia. Foi internado, e a tomografia de crânio evidenciou hidrocefalia tetraventricular com transudação ependimária importante (-Fig. 1). Após exame de líquor sem evidências de infecção, foi realizado implante de

Copyright $\odot 2015$ by Thieme Publicações License terms Ltda, Rio de Janeiro, Brazil
DOI http://dx.doi.org/ 10.1055/s-0035-1564822. ISSN 0103-5355. 


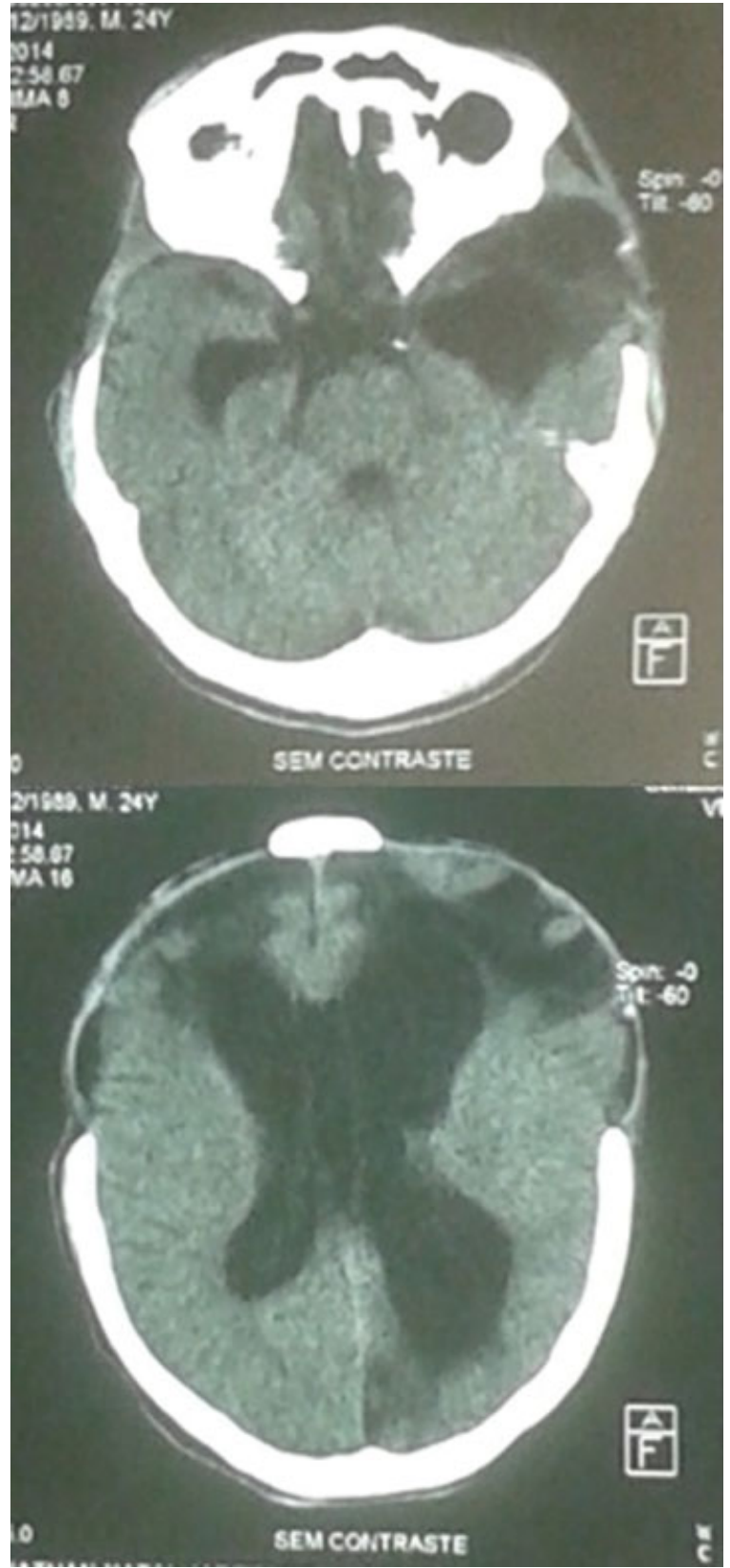

Fig. 1 Hidrocefalia tetraventricular com transudação ependimária importante.

derivação ventriculoperitoneal (DVP) ( - Fig. 2). No pós-operatório, o paciente apresentava boa evolução, com escala de coma de Glasgow (ECG) igual a 11. Porém, uma semana após a DVP, ele cursou com piora neurológica importante, com rebaixamento do sensório, ECG igual a 3, e depressão da craniolacuna.

No entanto, apresentava razoável padrão respiratório em ar ambiente, sugerindo-se a hipótese de síndrome do trefinado (-Fig. 3).

Dessa maneira, realizou-se cranioplastia externa (com capacete de gesso), melhorando o quadro neurológico no dia seguinte. Subsequentemente, o paciente foi submetido à ligadura da DVP, com proposta de reabertura após cranioplastia, realizando-se apenas observação do quadro. Assim, o

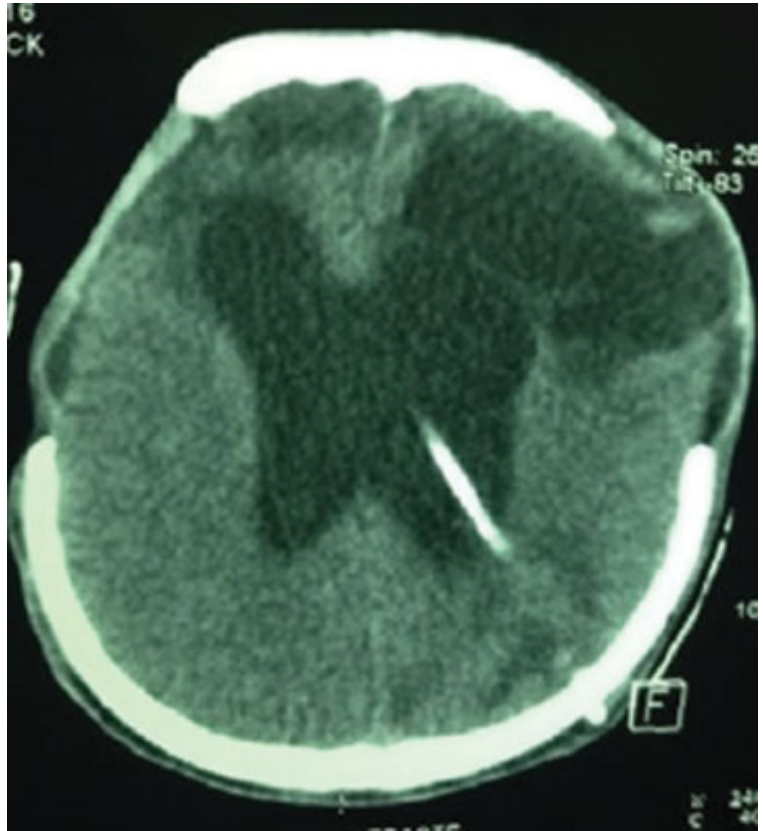

Fig. 2 Pós-operatório de DVP.

paciente foi submetido à cranioplastia heteróloga bifrontal com metilmetacrilato, em ato sem intercorrências, com melhora do quadro.

Após 10 dias, o paciente evoluiu com rebaixamento de sensório e formação de coleção sob a pele, levantando-se a hipótese de disfunção de válvula. A tomografia de crânio mostrou higroma subdural à direita e coleção hemorrágica à esquerda, e optou-se por tratamento conservador. (-Fig. 4). Poucos dias depois, o paciente apresentou drenagem de secreção purulenta pela ferida operatória.

No exame físico, o paciente mostrava-se sonolento, despertando a estímulo doloroso e localizando a dor bilateralmente, com pupilas $3+/ 3+$. Houve ainda deiscência da ferida operatória com secreção purulenta de odor fétido,

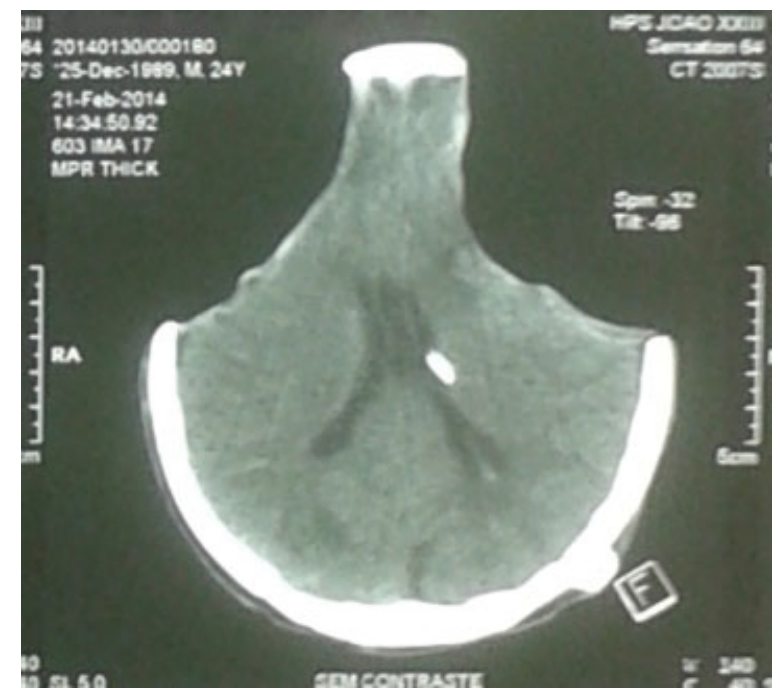

Fig. 3 Depressão de craniectomia bilateral. 


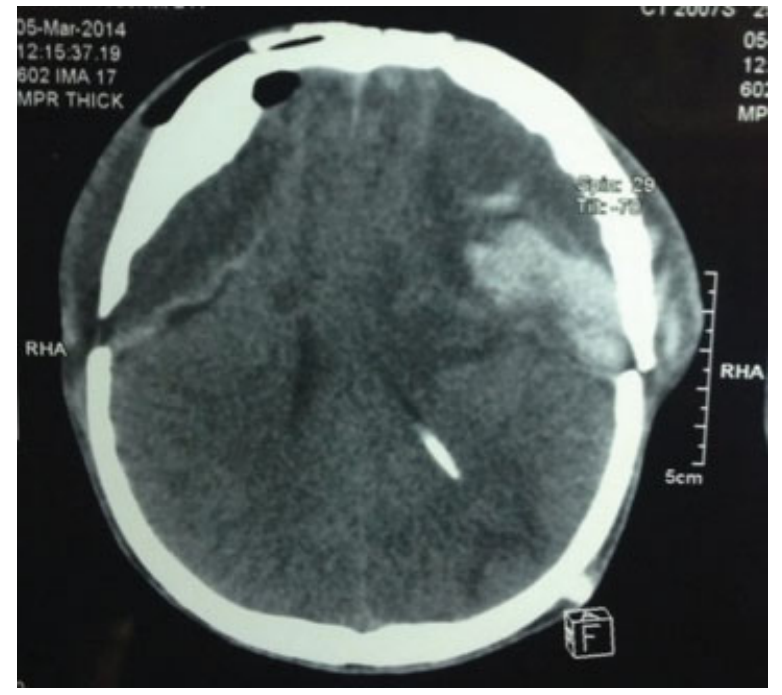

Fig. 4 Higroma subdural à direita e coleção hemorrágica à esquerda.

sendo sugerida infecção em sítio de cranioplastia. A conduta foi a retirada da cranioplastia, desbridamento do sítio da cranioplastia, acompanhada de tratamento com antibiótico (vancomicina e amicacina) e drenagem do empiema extradural. Além disso, houve a retirada da DVP e colocação de derivação ventricular externa (DVE), a qual foi monitorada no pós-operatório, necessitando de abertura constante durante a evolução.

Após controle da infecção, o paciente foi submetido à DVP de alta pressão à direita, com posterior retirada da DVE parietal esquerda.

A evolução neurológica do paciente foi estável, com janela da craniectomia abaulada, mas deprimível ou não tensa. Referente ao quadro neurológico, o paciente apresentavase alerta, localizava dor e emitia sons incompreensíveis.

Atualmente, convive com quadro sequelar funcional moderado.

\section{Discussão}

Yamaura e Makina ${ }^{3}$ definiram a síndrome do trefinado como alterações neurológicas que poderiam ser explicadas unicamente devido à concavidade do retalho de pele e à pressão da atmosfera sobre o tecido cerebral subjacente.

Outros investigadores têm procurado explicar a fisiopatologia desse fenômeno. Uma teoria seria de que a pressão atmosférica a ser transmitida diretamente para a cavidade intracraniana deslocaria o couro cabeludo ao longo do sítio de craniectomia para o interior. ${ }^{4}$

Recentemente, vários autores ${ }^{5,6}$ propuseram que um gradiente negativo entre a pressão atmosférica e intracraniana, que é agravada por alterações no compartimento do líquido cefalorraquidiano (LCR), seria o mecanismo de deterioração neurológica após craniectomia.

A drenagem do LCR em um paciente que sofre de hidrocefalia e meningite exacerba este efeito por meio da criação de um gradiente de pressão através do sítio de craniectomia.
Além disso, desidratação prolongada e posição ortostática podem precipitar esse fenômeno. ${ }^{7}$

A fisiopatologia alterada encontrada em um crânio convertido a partir de uma "caixa fechada" para uma "caixa aberta" traz benefícios e riscos, como no caso relatado. Em alguns pacientes que realizaram craniectomia, as forças da pressão atmosférica e da gravidade sobrecarregam a pressão intracraniana, consequentemente o cérebro parece afundado. Isto pode levar à hérnia de paradoxal e à síndrome do trefinado, que é uma emergência neurológica. 2,7-9

A craniectomia descompressiva é frequentemente realizada em caráter de urgência no cenário de pressões intracranianas incontroláveis a partir de uma variedade de causas. Os relatos iniciais focam seu benefício na definição das massas ou edema cerebral localizado. No entanto, as indicações estão se expandindo e agora incluem: lesão cerebral traumática com pressões refratárias intracranianas, hematomas subdurais, inchaço cerebral por vasoespasmo após hemorragia subaracnóidea, encefalite, sangramentos ou hematomas hipertensos intracerebrais, trombose venosa cerebral e acidentes vasculares encefálicos. ${ }^{2}$ No entanto, este procedimento pode levar à síndrome do trefinado que deve ser suspeita quando houver deterioração neurológica após craniectomia, especialmente quando a pele do sítio de craniectomia é afundada. ${ }^{10}$

De acordo com Shirley et al, ${ }^{11}$ outras complicações são:

$\rightarrow$ Complicações perioperatórias

- Expansão hemorrágica de contusões é inerente ao processo de lesão e tem sido demonstrada em tomografias computadorizadas em pacientes com TCE.

- Craniectomia descompressiva para TCE pode levar a uma nova lesão em massa, contralateral ou remota, para o hemisfério descomprimido.

- Expansão do cérebro com hérnia cerebral externa através do defeito da craniectomia é frequentemente observada no início do período após a descompressão (-Fig. 5).

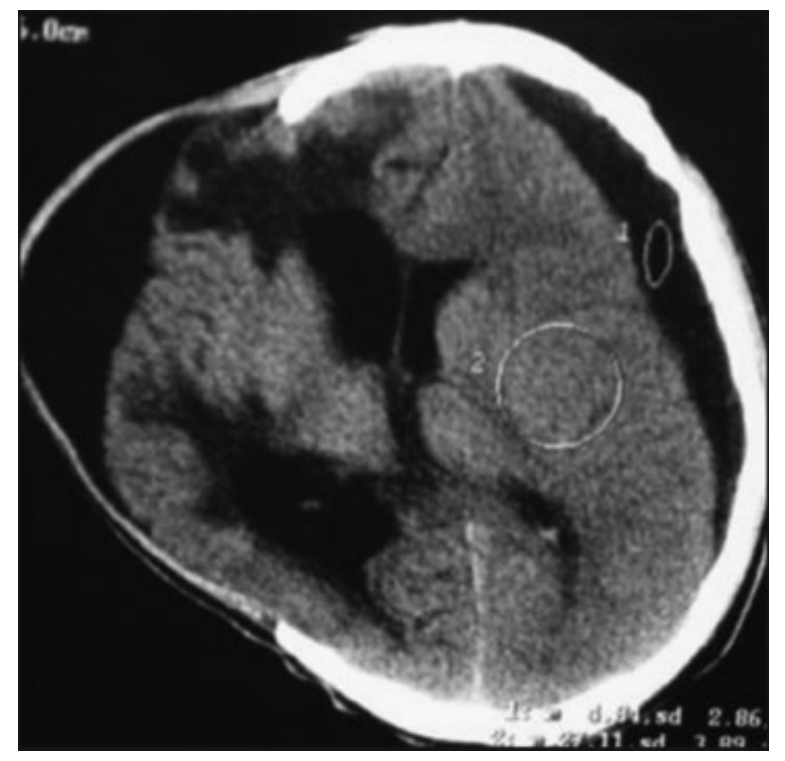

Fig. 5 Higroma subdural associado à herniação cerebral externa. 
$\rightarrow$ Complicações pós-operatórias (dentro de 30 dias)

- Higromas subdurais: craniectomia altera a dinâmica da circulação do LCR (-Fig. 5 ).

- Hérnia paradoxal, com compressão do tronco cerebral e deterioração neurológica, pode apresentar-se de forma atrasada após uma punção lombar em pacientes com a craniectomia descompressiva.

$\rightarrow$ Complicações tardias, após 1 mês

- Hidrocefalia e síndrome do trefinado são as complicações mais frequentes da craniectomia descompressiva após 1 mês. Além disso, foi identificada como fator de risco para alterações no LCR e desenvolvimento de hidrocefalia póstraumática.

- Infecções.

- Na craniectomia descompressiva, a reabsorção de retalhos ósseos livres é comum e pode aproximar-se de uma incidência tão alta quanto $50 \%$ no acompanhamento a longo prazo.

- Estado vegetativo persistente.

O objetivo do tratamento do paciente com a síndrome do trefinado é a restauração da pressão exercida pela depressão do sítio de craniectomia. ${ }^{7}$

Segal et al $^{12}$ sugeriram que a cranioplastia melhora os déficits neurológicos por uma diminuição da pressão intracraniana local e correção da dinâmica anormal do LCR. No entanto, a cranioplastia para grave defeito no crânio pode resultar em disfunção do cérebro subjacente, risco de formação de coleção líquida e de formação de hematoma no espaço subdural, devido ao grande espaço morto. ${ }^{7}$ Os métodos cirúrgicos seguros e eficazes para expandir a depressão no couro e para eliminar o espaço morto no contexto do shunt ventrículo-ponte são: oclusão temporária ou remoção do dispositivo de derivação antes da cranioplastia. ${ }^{13}$

Fodstad et al $^{14}$ propuseram que apenas sintomas aliviados por cranioplastia devem ser incluídos na definição de síndrome do trefinado. Outros sintomas associados a esta síndrome são: queixas subjetivas, hemiparesia, defeitos no sensório, disfasia, convulsões, síndrome do mesencéfalo, disfunção mesodiencefálica e mutismo acinético.

\section{Conclusão}

A compreensão aprofundada da síndrome do trefinado se constitui relevante, uma vez que é uma das temíveis complicações da craniectomia descompressiva.

O tratamento da ST com a cranioplastia temporária (calota de gesso externa) tem mostrado grandes resultados, e seu efeito imediato ajuda na diminuição dos efeitos pressóricos externos e na programação da cranioplastia definitiva.

Portanto, a cranioplastia deve ser realizada o mais precocemente possível, diminuindo as chances de complicações da craniectomia descompressiva, tal como a síndrome do trefinado, melhorando a sobrevida do paciente e auxiliando na recuperação dos danos neurológicos.

Conflitos de Interesse

Os autores declaram não haver conflitos de interesse.

\section{Referências}

1 Joseph V, Reilly P. Syndrome of the trephined. J Neurosurg 2009; 111(4):650-652

2 Akins PT, Guppy KH. Sinking skin flaps, paradoxical herniation, and external brain tamponade: a review of decompressive craniectomy management. Neurocrit Care 2008;9(2):269-276

3 Yamaura A, Makino H. Neurological deficits in the presence of the sinking skin flap following decompressive craniectomy. Neurol Med Chir (Tokyo) 1977;17(1 Pt 1):43-53

4 Langfitt TW. Increased intracranial pressure. Clin Neurosurg 1969;16:436-471

5 Oyelese AA, Steinberg GK, Huhn SL, Wijman CA. Paradoxical cerebral herniation secondary to lumbar puncture after decompressive craniectomy for a large space-occupying hemispheric stroke: case report. Neurosurgery 2005;57(3):E594, discussion E594

6 Schwab S, Erbguth F, Aschoff A, Orberk E, Spranger M, Hacke W. ["Paradoxical" herniation after decompressive trephining]. Nervenarzt 1998;69(10):896-900

7 Han PY, Kim JH, Kang HI, Kim JS. "Syndrome of the sinking skinflap" secondary to the ventriculoperitoneal shunt after craniectomy. J Korean Neurosurg Soc 2008;43(1):51-53

8 Romero FR, Zanini MA, Ducati LG, Gabarra RC. Sinking skin flap syndrome with delayed dysautonomic syndrome-An atypical presentation. Int J Surg Case Rep 2013;4(11): 1007-1009

9 Gadde J, Dross P, Spina M. Syndrome of the trephined (sinking skin flap syndrome) with and without paradoxical herniation: a series of case reports and review. Del Med J 2012;84(7):213-218

10 Chalouhi N, Teufack S, Fernando Gonzalez L, Rosenwasser RH, Jabbour PM. An extreme case of the syndrome of the trephined requiring the use of a novel titanium plate. Neurologist 2012; 18(6):423-425

11 Stiver SI. Complications of decompressive craniectomy for traumatic brain injury. Neurosurg Focus 2009;26(6):E7

12 Segal DH, Oppenheim JS, Murovic JA. Neurological recovery after cranioplasty. Neurosurgery 1994;34(4):729-731, discussion 731

13 Liao CC, Kao MC. Cranioplasty for patients with severe depressed skull bone defect after cerebrospinal fluid shunting. J Clin Neurosci 2002;9(5):553-555

14 Fodstad H, Love JA, Ekstedt J, Fridén H, Liliequist B. Effect of cranioplasty on cerebrospinal fluid hydrodynamics in patients with the syndrome of the trephined. Acta Neurochir (Wien) 1984; $70(1-2): 21-30$ 